\title{
Nahrungsbeziehungen der Turbellarien in Küstensalzwiesen ${ }^{1}$
}

\author{
Martin Bilio ${ }^{2}$ \\ Hydrobiologisch Institunt, Afd. Delta-Onderzoek \\ Yerseke, Niederlande
}

\begin{abstract}
Food relations of turbellarians in salt marshes. Turbellarians represent one of the most characteristic and often also most numerous elements of the salt-marsh biocoenosis. The analysis of their food relations promised, therefore, insights into the food web in salt marshes. Two questions are of particular importance: (1) Is autochthonous or allochthonous food more important? (2) Are there noticeable relations to the aerial phase of the biotope? Gut-content surveys, feeding-experiments and information from literature indicate, that the main food of salt-marsh turbellarians consists of oligochetes, nematodes and diatoms. There exist certain relations between the taxonomic categories of turbellarians and their food sources, and, furthermore, between the type of pharynx and the kind of food. Oligochetes are 4 and nematodes up to 14 times more abundant than turbellarians. Nematodes are also likely to be the most important food competitors for turbellarians. Enemies of salt-marsh turbellarians are the polychaete Nereis diversicolor and sporozoan parasites. The main food of salt-marsh turbellarians consists of organisms existing in sufficient numbers in the biotope; a dependency on allochthonous food is therefore unlikely. Allochthonous food (e. g. copepods from adjacent mud flats) may, however, at times enlarge the food resources available. Little evidence for the use of air-living organisms as food, and no evidence for air-living enemies, was found. The aquatic food chain in salt marshes ends at the level of flatworm-eating polychaetes or, where these are absent, already at that of the turbellarians themselves. Although the biomass (standing crop) of primary producers reaches its maximum in the most protected part of the beach (salt marshes), maximum population density of turbellarians is found in parts of maximum exposure. This fact is considered to be due to the great amount of allochthonous food available at the upper edge of the surf zone.
\end{abstract}

\section{EINLEITUNG}

Die ökologische Eigenart der Uferbiotope ergibt sich aus der Grenzsituation zwischen den beiden großen Lebensräumen Wasser und Land. Je nachdem, wie weir aquatische und terrestrische Milieubedingungen einander durchdringen, äußert sich die Randlage in einer mehr oder weniger starken Verzahnung zwischen der Flora und Fauna des Wassers einerseits und der des Landes andererseits. Daneben können aus dem Zusammenspiel der gegensätzlichen Faktoren spezielle Uferhabitate resultieren,

1 Herrn Professor Dr. Alexander Luther, Helsingfors, zum 90. Geburtstag am 17. Februar 1967 in Verehrung und Dankbarkeit gewidmet.

2 Neue Anschrift: Euratom - C.N.E.N., Fiascherino (La Spezia), Italien. 
die die Existenz spezifischer Uferorganismen ermöglichen. Innerhalb der aquatischen Bodenfauna der Küstensalzwiesen stellen die Turbellarien einen hohen Anteil solcher charakteristischer Uferbewohner (BILIO 1964 b, 1966 b), und es liegt nahe, danach zu fragen, ob die enge Bindung an das besondere Milieu auch in ihren Nahrungsbeziehungen zum Ausdruck kommt.

$\mathrm{Zu}$ den charakteristischen Milieubedingungen der Uferbiotope gehört die Zufuhr von Substrat- und Nahrungsmaterial aus dem Wasser und auch aus anderen Uferbezirken. Diese Nahrungsquelle kann für manche Uferorganismen die Hauptgrundlage ihrer Existenz sein. Man denke an die „wrack-fauna“ - die Bewohner des Strandanwurfs! Die Frage nach den Nahrungsbeziehungen der Salzwiesenturbellarien ist also auch unter diesem Gesichtspunkt zu erörtern.

Die Küstensalzwiesen bilden den Abschnitt des Meeresufers, in dem die allgemeinen trophischen Bedingungen am günstigsten sind. Während am Brandungsstrand die Ablagerung von mineralischem und organischem Feinmaterial und die Entstehung einer geschlossenen Vegetationsschicht durch die starke Wasserbewegung weitgehend verhin. dert werden, können am Stillwasserufer große Mengen feiner Sinkstoffe sedimentieren und perennierende Gefäßpflanzen sich zu einer geschlossenen Decke verbinden. Hier erreicht also die Biomasse der Primärproduzenten ihre höchsten Werte.

Das Salzwiesenufer wird im Bereich der Nord- und Ostsee von mindestens 50 biotopeigenen und biotopverwandten Turbellarienarten besiedelt, die vornehmlich zu den Neorhabdocoela (35 Arten) sowie den Seriata (6 Arten) und den Macrostomida (5 Arten) gehören. Zwei Arten sind Vertreter der Catenulida, und je eine Art stellen die Prolecithophora und die Acoela (vgl. Bilio 1966 b, p. 311, Tabelle 1). Diese Zahlen sind Ergebnisse von Untersuchungen in deutschen, finnischen und niederländischen Küstenbezirken ${ }^{3}$.

\section{NAHRUNGSOB JEKT'E}

Uber die Nahrungsbeziehungen ganzer Lebensgemeinschaften von Turbellarien wissen wir noch wenig. Ein vollständiges Bild dieser Verhältnisse in den Salzwiesen können auch die folgenden Ausführungen nicht vermitteln. Es erscheint aber möglich und für die weitere Arbeit sinnvoll, einige schon jetzt deutliche Züge aufzuzeichnen und gewisse Probleme anzuschneiden. Die den folgenden Erörterungen zugrunde liegenden Daten stammen zu einem erheblichen Teil aus der eigenen, in erster Linie auf ökologische Ziele abgestimmten Determinationsarbeit, zum anderen aus der Literatur. Systematische Fütterungsversuche wurden mit der maricolen Triclade Uteriporus vulgaris angestellt (vgl. Bitro 1964 b, p. 536). Einzelexperimente galten auch anderen Arten.

Was kommt für die Salzwiesenturbellarien als Nahrung in Frage, und welche Objekte sind tatsächlich als Nahrung bekannt? In Betracht gezogen werden müssen alle organischen Substratbestandteile, die in den Darm aufgenommen werden können,

3 Im Gegensatz zu den unter anderen Gesichtspunkten zusammengestellten Zahlen (Brio 1966b) sind hier die poly- und mesohalinen Bereiche des südwestniederländischen Delta-Gebietes mitberücksichtigt. 
und alle Organismen, die im Lebensraum entweder tot vorgefunden oder lebend bewältigt werden können. Wir gehen dabei zunächst davon aus, welche Angaben in der Literatur für Turbellarien zu finden sind, beschränken uns aber bei unseren Schlußfolgerungen auf die an Salzwiesenturbellarien gemachten Beobachtungen.

Sortiert man größere Mengen Turbellarien lebend aus Substratproben und bestimmt sie anschließend im Quetschpräparat, so hat man vor allem die Möglichkeit, den Darminhalt zu kontrollieren. Außerdem kann man hin und wieder auch direkte Beobachtungen über die Nahrungsaufnahme machen. Eine wesentliche Fehlerquelle der Darminhaltskontrolle liegt in der unterschiedlichen Zersetzbarkeit und Zersetzungsdauer der Nahrungsobjekte. Nahrungsorganismen mit widerstandsfähigen Kutikularstrukturen sind im Darm länger nachweisbar als reine Weichkörper beziehungsweise nur sehr schwach strukturierte Nahrung. Diatomeenschalen, die Borsten von Oligochaeten, die Spicula männlicher Nematoden und die Hartteile des Kauapparates von Rotatorien werden daher sicher öfter beobachtet als etwa Ciliaten und vor allem Bakterien. Ein weiterer Fehler, dessen wir uns auch bei der folgenden Übersicht bewußt bleiben müssen, kann bei ausschließlichem Aussaugen von Nahrungsorganismen auftreten, weil dann oft gerade die schwer zersetzbaren Strukturen (Kutikula von Crustaceen und Insektenlarven) gar nicht erst in den Darm gelangen.

Detritus wird in der Literatur hin und wieder als Turbellarien-Nahrung angegeben (z. B. Westblad 1923, p. 54), für Salzwiesen-Turbellarien ist er jedoch bisher nicht als wesentliche Nahrungsgrundlage bekannt. Da ein sicherer Nachweis von Detritus als Nahrung schwierig ist, wird er als mögliche Nahrung allgemein in Rechnung gestellt. (Detritus kann unter anderem auch als Darminhalt von detritusfressenden Beutetieren in den Darm von Turbellarien gelangen.) In der gleichen Weise müssen wir mangels einwandfreier Beobachtungen $B$ a $\mathrm{k} t$ e $\mathrm{r}$ i e $\mathrm{n}$ berïcksichtigen.

Häufig findet man im Darm von Turbellarien D i a to me en. Von den Salzwiesenturbellarien sind vor allem die Vertreter der Gattungen Baicalellia, Provortex und Vejdovskya unter den Neorhabdocoela und der Gattung Macrostomum unter den Macrostomida zu erwähnen. Aber auch im Darm von Mecynostomum sp. ${ }^{4}$, dem einzigen Repräsentanten der Acoela, sowie von Vertretern der Monocelididae (Seriata) und manchmal auch der Proxenetidae (Neorhabdocoela) sind Diatomeen festgestellt worden. Bei den Proxenetiden scheint es sich - von nur wenigen bisher erkennbaren Ausnahmen abgesehen - eher um eine Zusatz- oder Notnahrung zu handeln. Ausschließlich und zahlreich wurden Diatomeen im Darm von Baicalellia brevitubus gefunden (Luther 1962, Bilio 1964b; außerdem 15 neue Beobachtungen). Dieses Turbellar kann sich so mit Diatomeen vollstopfen, daß es dadurch seine normale Körperform verliert und wie verunstaltet aussieht. Nur Diatomeen sind bisher ferner im Darm von Provortex pallidus (Luther 1948) und P. karlingi (LutHer 1962), Vejdovskya pellucida (Tafel IV, Fig. 5 bei Schultze 1851) und Westbladiella obliquepharynx (Luther 1948; 3 neue Beobachtungen) und von Archimonotresis limophila

4) Zusatz während der Korrektur: Nach brieflicher Mitteilung von Professor Dr. P. Ax, Göttingen, wurde die in den Salzwiesen lebende Mecynostomum-Art von Dr. J. DörJes, Wilhelmshaven, inzwischen als Mecynostomum auritum (M. ScHulTZE 1851) identifiziert. Sie ist mit der von LUTHER (1960) unter diesem Namen publizierten Form identisch, nicht jedoch mit M. auritum in der Bearbeitung durch Westblad $(1946,1948)$ (vgl. auch Bilio 1964b, p. 527). 
(Westblad 1955), dem einzigen Vertreter der Prolecithophora, wahrgenommen worden.

„Algen“ und Cyanophyceen als Darminhalt gibt Luther (1960) für Macrostomum balticum beziehungsweise $M$. tenuicauda an. G $\mathrm{r}$ ü n e A $1 \mathrm{~g}$ e $\mathrm{n}$ z ell e n mit rotem Pigmentfleck wurden bei Macrostomum bystricinum (5 Beobachtungen), bei M. tenuicauda (1 Beobachtung) und vor allem bei Vejdovskya ignava (19 Beobachtungen) gefunden. V. ignava ist ein typischer Vertreter der Canophyceensand-Fauna, der von hier aus in die Salzwiesen gelangt.

Eine Übersicht über die wichtigsten Tiergruppen, die neben den Turbellarien im Salzwiesenboden vorkommen, vermittelt Tabelle 1. P r o t o z o e n sind im Darm von Turbellarien dann gut nachzuweisen, wenn es sich um beschalte Formen handelt. Thekamoeben sind bei Macrostomum hystricinum (LuTHER 1960), M. hamatum (eigene

Tabelle 1

Wichtigste Tiergruppen der Begleitfauna

\begin{tabular}{|ll|}
\hline $\begin{array}{l}\text { Aquatische Fauna } \\
\text { Thekamoeben }\end{array}$ & Ubergangsgruppen \\
Foraminiferen & $\begin{array}{l}\text { Oligochaeten } \\
\text { Ciliaten }\end{array}$ \\
Dematoden & \\
Rotatorien & \\
Polychaeten & \\
Oligochaeten & \\
Halacariden & Aerische Fauna \\
Ostracoden & Oribatiden \\
Copepoden & Collembolen \\
\hline
\end{tabular}

Beobachtung) und $M$. tenuicauda (LuTHER 1960; eigene Beobachtung) festgestellt worden, und zwar ausschließlich in Brackwassergebieten, auf die auch die drei Turbellarien beschränkt sind. Foraminiferen hingegen, die die Salzwiesen sowohl in eu- und polyhalinen als auch in mesohalinen Gebieten in beträchtlicher Individuendichte besiedeln können, sind bisher nicht im Darm von Turbellarien gefunden worden. Ciliaten kommen als Nahrung in Frage. Die einzige in Salzwiesen nicht nur als Irrgast auftretende Art, in deren Darm Ciliaten beobachtet wurden, ist die schon als Algenfresser erwähnte Vejdovskya ignava (Luther 1962; 4 neue Beobachtungen), die übrigens auch Diatomeen aufnimmt.

Eine bedeutende Rolle für die Ernährung der Salzwiesenturbellarien spielen zweifellos die $\mathrm{Ne}$ m a to de n. Sie sind im Darm von zehn Arten festgestellt worden. Als wichtigste Nematodenfresser sind die Repräsentanten der Proxenetidae zu betrachten. Beobachtungen liegen für Proxenetes karlingi (Luther 1962), P. deltoides (Den HarTOG 1965 a, 1966 b), P. puccinellicola (DEN HARtog $1966 \mathrm{a}, \mathrm{b}$ ), P. cisorius (DeN Hartog 1966 a, b) und Ptychopera spinifera (3 neue Beobachtungen) vor. Im Darm von Proxenetes deltoides (Den Hartog 1965 a) und P. puccinellicola (Den Hartog 1966 a) wurden auch Diatomeen gefunden, für die kleine (nur 0,2-0,5 mm lange) Ptychopera spinifera führt Den Hartog nur Diatomeen an. Außerdem sind Nema- 
toden beziehungsweise Reste von Nematoden in Darm des Kalyptorhynchiers Placorbynchus octaculeatus (Neorhabdocoela), von Monocelis fusca (Seriata) und Macrostomum bystricinum gesehen worden. Bei $M$. bystricinum war es nur ein kleiner Nematode (eigene Beobachtung), bei Monocelis fusca fand Den Hartog (1964 a) Reste von Nematoden. Für Placorbynchus octaculeatus scheinen Nematoden ein Teil der Hauptnahrung zu sein (KarLING 1963; 4 neue Beobachtungen).

Rotatorien sind - von Bdelloiden in brackigen Gebieten und in höheren Salzwiesenzonen abgesehen - im Lebensraum relativ selten. Wahrgenommen wurden Formen dieser Tiergruppe im Darm von Mecynostomum sp. (KARLING, nach Luther 1960; gleichzeitig werden auch Gastrotrichen genannt), von Macrostomum hamatum (eigene Beobachtungen, in einem Fall befanden sich fünf Rotatorien gleichzeitig im Darm), M. tenuicanda (Luther 1960), Provortex balticus (Lu'rHer 1962), Thalassoplanella collaris (eigene Beobachtungen) und Acrorbynchides robustus (KarLING 1963).

Eine zweite Gruppe der Begleitfauna, die einen erheblichen Teil der Ernährungsbasis bildet, sind die O I g o ch a e te n - wie die Nematoden eine der individuenreichsten Tiergruppen der Salzwiesenfauna. Die Oligochaetenfresser unter den Salzwiesenturbellarien gehören fast alle zu den Seriata und zu den Typhloplanoida und Kalyptorhynchia unter den Neorhabdocoela. Ausnahmen sind - nach unserer gegenwärtigen, auch hier sicher noch unvollständigen Kenntnis - innerhalb der Salzwiesenfauna nur Pseudograffilla arenicola (Neorhabdocoela, Dalyellioida; zwei eigene Beobachtungen, Hauptnahrung: Turbellarien) und Macrostomum spirale (einmal Oligochaeten-Borsten im Darm gesehen, außerdem einmal ein Turbellar).

Mit Vorliebe Oligochaeten scheinen Prognathorbynchus canaliculatus (KARLrNG 1956; 7 neue Beobachtungen), Castrada subsalsa (LuTHER 1963, zahlreiche neue Beobachtungen) und Uteriporus vulgaris (Brio 1964 b) zu fressen. Von P. canaliculatus und C. subsalsa liegen bisher sogar nur einschlägige Beobachtungen vor, Uteriporus nahm bei Fütterungsversuchen auch Schmetterlingsraupen an (Brio 1964 b, p. 536). Nur Borsten von Oligochaeten wurden ferner im Darm je zweier Individuen von Parautelga bilioi und Coronbelmis multispinosus bemerkt (eigene Beobachtungen) und einmal in einem Exemplar von Minona baltica (Karling, nach LUTHer 1960). Oligochaeten neben anderen Organismen dienen Monocelis lineata (4 eigene Beobachtungen) und Coelogynopora schulzii (Brto 1964 b, ferner 3 neue Beobachtungen), Proxenetes deltoides (Den Hartog 1965 a, 1966 b), P. puccinellicola und $P$. cisorins (beide DeN HARTog 1966 a) als Nahrung. Im Darm von Archilopsis unipunctata wurden bisher nur Diatomeen sicher nachgewiesen (DEN HARTOG 1964 a); noch nicht geschlechtsreife Exemplare einer Monocelidide ohne Augenfleck und ohne dunkles Körperpigment, die im Früjahr 1966 am unteren Rand einer relativ exponierten Salzwiese bei Tvärminne (finnische Ostseeküste) gefunden wurden, hatten Oligochaeten-Borsten im Darm. Die einzige Art der Gattung Proxenetes, in deren Darm bisher nur Oligochaeten-Reste festgestellt wurden, ist $P$. britannicus (DEN HARTog 1966 a).

Nach den Oligochaeten bleiben unter der aquatischen Begleitfauna nur noch die Arthropoden und die Turbellarien selbst als mögliche Nahrungsobjekte übrig. $\mathrm{H}$ a 1 a c a rid e n oder Reste von Halacariden wurden noch gar nicht im Darm von Salzwiesenturbellarien beobachtet, Ostracoden nur bei Macrostomum hystricinum (einmal ein kleines Exemplar, Luther 1960). Co p e pod e n werden als Nahrungs- 
objekte von Acrorbynchides robustus (KARLING 1963, neben Rotatorien, vgl, obige Ausführungen) angegeben. Direkt beobachten konnte ich den Angriff und die Überwältigung eines Copepoden durch Thalassoplanella collaris. Der Copepode wurde nur ausgesaugt und auch nur teilweise. $\mathrm{Da}$ Copepoden - insbesondere Harpacticoidea - in den Salzwiesen nicht selten sind, müssen sie angesichts dieser Beobachtung in höherem Maße als Turbellariennahrung in Rechnung gestellt werden als bei ausschließlicher Wertung von Wahrnehmungen am Darminhalt.

Besonders interessant - im Hinblick auf die Diskussion der den Turbellarien übergeordneten trophischen Stufe - sind die Turbellarien, die sich von anderen T u r b e 1 1 a r i e n ernähren. Zwei Arten stehen hier vornean: Pseudograffilla arenicola als be-

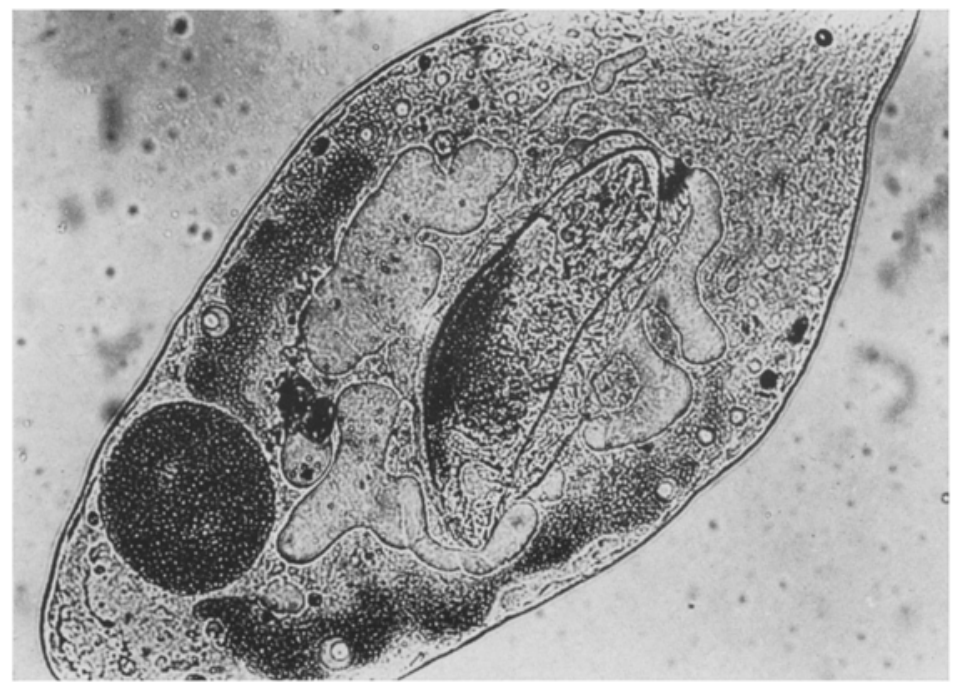

Abb. 1: Mecynostomum sp. mit einverleibtem Exemplar von Placorbyncbus octaculeatus dimorphis Karing 1947 (Bildmitte). Oben rechts (nahe dem oberen Bildrand) die Statocyste, ganz links nahezu ablagereifes Ei der Acoelen; Vorderende des Kalyptorhynchiers auf den unteren Bildrand gerichtet. (Tvärminne, 21. August 1963)

sonders großer Vertreter der Dalyellioida (bis 2,5 mm lang und von sehr gedrungenem Habitus; s. auch weiter oben), und Mecynostomum sp., die einzige (schon bei Diatomeen und Rotatorien erwähnte) Acoele in den Salzwiesen.

In Mecynostomum sp. habe ich viermal ganze Turbellarien feststellen können, darunter die Arten Placorbynchus octaculeatus (ssp. dimorphis; s. Abb. 1), Ptychopera westbladi und Proxenetes bilioi. Von den beiden Proxenetiden wurde außerdem je einmal nur das Stilett im Körper von Mecynostomum gesehen, und ferner war in zwei Fällen eine fremde, gelbliche Eikapsel aufgenommen worden. Über Turbellarien als Beute von Pseudograffilla arenicola wurde schon früher berichtet (LuTHER 1948, Bılı 1964 b); ferner wurde jetzt einmal das Stilett von Baicalellia brevitubus gefunden. Daß Turbellarien einen Hauptteil der Nahrung dieser Art ausmachen, dürfte nach den vorliegenden Beobachtungen kaum zu bezweifeln sein. 
Turbellarien werden auch als Teil der Nahrung von Placorbynchus octaculeatus („andere Kleinturbellarien“, Karling 1963) und von Provortex balticus (eine Acoele, LuTHER 1962) angegeben. Unter den Exemplaren von $P$. balticus, die mir selbst untergekommen sind, hatten zwei je eine junge Proxenetide verschluckt. $P$. balticus ist unter den in Salzwiesen vorkommenden Dalyellioiden nächst Pseudograffilla der größte Vertreter dieser Verwandtschaftsgruppe! Eine junge Neorhabdocoele (vermutlich Parawtelga bilioi) fand sich einmal auch im Darm von Macrostomum spirale, und schließlich sind je ein Stilett von Promesostoma marmoratum und Macrostomum cf. balticum in Monocelis fusca (Den Hartog 1964 a bzw. eigene Beobachtung an einem juvenilen Exemplar) und ein Stilett von Macrostomum spirale in Monocelis cf. lineata (juveniles Exemplar, eigene Beobachtung) entdeckt worden.

Beziehungen zu terrestrischen Arthropoden-Gruppen (vgl. Tabelle 1, Übergangsgruppe Dipteren-Larven und die aerische Fauna) sind mit Ausnahme der maricolen Triclade Uteriporus vulgaris, die im Experiment Lepidopteren-Larven annimmt, bisher für die Turbellarien der Salzwiesenfauna nicht nachgewiesen. Vorsichtig machen muß uns außer den Versuchen an Uteriporus auch eine Beobachtung von LUTHER (1948, p. 85, und 1962, p. 61), der unter anderem schildert, wie eine Promesostoma marmoratum ${ }^{1}$ an einer sehr viel größeren Chironomidenlarve saugte. Auch Dipteren L a rven sind regelmäßige Bewohner von Salzwiesen, und auch mit ihnen als Nahrung von Salzwiesenturbellarien müssen wir also rechnen.

\section{LEBENSFORMTYPEN}

Resumieren wir diese Fakten noch nach einigen allgemeinen Gesichtspunkten, ehe wir zu den Fragen nach der Herkunft der Nahrung übergehen. Es fällt auf, daß gewisse Beziehungen zwischen der systematischen Stellung der Turbellarien und ihrer Vorzugsnahrung beziehungsweise zu ihrem Nahrungsspektrum bestehen, so vor allem zwischen den Proxenetiden und den Nematoden. Beziehen wir in die Betrachtung dieser Verwandtschaftsgruppe, von der unsere Kenntnis erst kürzlich durch die Publikationen von DeN HARToG wieder erheblich erweitert worden ist, auch die nicht zur Salzwiesenfauna gehörenden Arten der Gattungen Proxenetes und Ptychopera ein, so wird das Bild noch deutlicher: Nach DeN HARTOG (1964 b-1966 b) umfassen diese beiden Gattungen heute insgesamt 24 Arten, an 13 davon sind bisher Nahrungsbeobachtungen gemacht worden, und bei 10 von diesen 13 Arten sind Nematoden als Nahrung festgestellt worden! Nur Nematoden konnten bisher für Ptychopera westbladi (DEN Hartog $1964 \mathrm{~b}$ und eigene Beobachtungen), Proxenetes karlingi (Luther 1962), P. flabellifer (Den Hartog 1965 a), P. simplex und P. bilioi (für beide Arten eigene Beobachtungen) registriert werden. Bei fünf Arten sind neben Nematoden auch andere Organismen als Nahrungsobjekte beobachtet worden, und zwar Oligochaeten, Diatomeen und Reste von "anderen“ Tieren bei Proxenetes puccinellicola, Oligochaeten und Diatomeen bei Proxenetes deltoides und P. quadrispinosus, nur Oligochaeten bei P. cisorius und nur Diatomeen bei Ptychopera spinifera (vgl. Den Hartog $1964 \mathrm{~b}$, 1965 a, b, 1966 a, b). Ausschließlich Bestandteile anderer Organismen sind bisher im

\footnotetext{
1 Diese Art kommt jedoch nur als Irrgast in den Salzwiesen vor.
} 
Darm von Proxenetes britannicus (Oligochaeten), P. trigonus (bei einem Exemplar Reste eines Copepoden, Den Hartog 1966 a) und $P$. inflatus (einmal eine teilweise verdaute Nemertine, nach DeN HARTog 1966 a) gesehen worden.

Ein weiterer Zug in dieser Richtung scheint mir das Überwiegen von Diatomeen und Oligochaeten und das Fehlen der Nematoden bei den Vertretern der Seriata zu sein. Ferner stellen Diatomeen und andere Einzeller offenbar die Hauptnahrung der kleinen Dalyellioiden dar, während sie bei den Kalyptorhynchiern bisher praktisch überhaupt nicht zu verzeichnen sind. KarLING (1963) gibt für Placorbynchus octaculeatus zwar auch Diatomeen an, vermerkt aber, daß sie von ausgesaugten Beutetieren

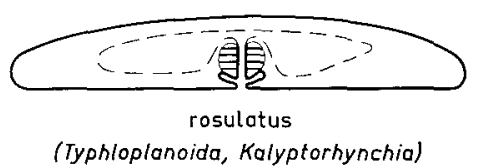

(Typhloplanoida, Kalyptorhynchia)

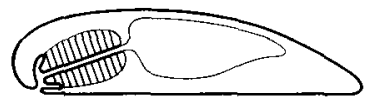

doliiformis

(Dalyellioida)
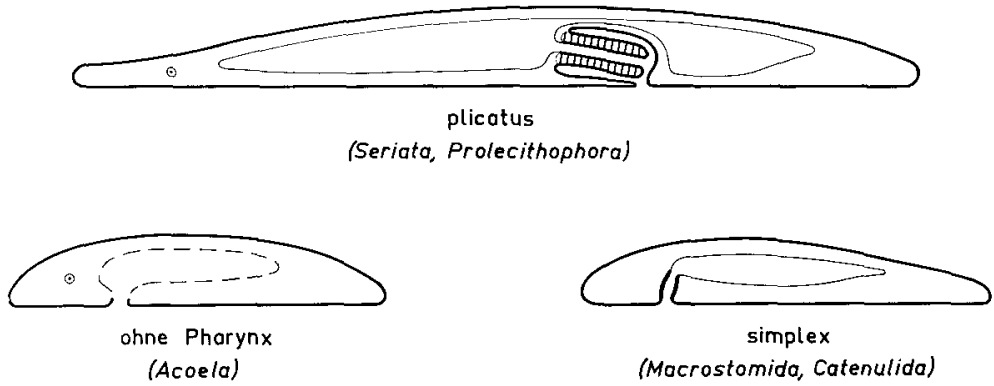

Abb. 2: Pharynxtypen der Salzwiesen-Turbellarien im Längsschnitt. Das Schema für die Acoela (links unten) bezieht sich auf Mecynostomum sp., links von der Mundöffnung die Statocyste. Das Statocystensymbol im Schema für den Pharynx plicatus gilt nur für die Proseriata. Der Pharynx plicatus des einzigen in Salzwiesen vorkommenden Vertreters der Prolecithophora (Archimonotresis limopbila) ist nach vorn gerichtet; ob auch für ihn die im Text gegebene Charakteristik gilt, ist fraglich. Die Unterbrechung der Umgrenzungslinie für den Darmbereich (links oben und unten) soll die Bildung eines Endocytiums andeuten (vgl. Ax 1961, p. 14)

herrühren könnten. Die Macrostomiden scheinen noch weniger auf bestimmte Nahrungsobjekte spezialisiert zu sein als die anderen Gruppen, und die einzige Acoele und die größte Dalyellioide sind vornehmlich Turbellarienfresser.

In diesem Zusammenhang liegt es nahe, die für die verschiedenen systematischen Gruppen charakteristischen Pharynxtypen als allgemeinere Beurteilungskriterien in Betracht zu ziehen. Schon Westrlad (1923, pp. 55-56) hat Hinweise in dieser Richtung gegeben. Seine Vermutung allerdings, der Pharynx rosulatus diene ausschließlich zum Aussaugen, nicht auch zum Verschlingen ganzer Beuteorganismen, läßt sich nicht bestätigen. Aus unserer Zusammenstellung der bis heute vorliegenden Nahrungsbeobachtungen an Salzwiesenturbellarien ergibt sich die folgende Übersicht (vgl. Abb. 2):

(a) Die pharynxlose Acoele Mecynostomum sp. kann sowohl die hartschaligen Diatomeen in den Körper aufnehmen als auch die weichhäutigen Turbellarien, dagegen 
anscheinend nicht die (zu sperrigen?) Nematoden. (b) Der Pharynx simplex der Macrostomida scheint (abgesehen davon, daß er kaum zum Aussaugen von Beuteorganismen befähigen dürtte und die Nahrungsobjekte daher im allgemeinen nicht wesentlich gröBer sein werden als der Konsument) zu keiner deutlichen Spezialisierung zu zwingen. (c) Der Pharynx plicatus läßt sich in erster Linie als Saug- und Schlürfpharynx charakterisieren (Oligochaeten, Diatomeen; Aas). (d) Der Pharynx rosulatus eignet sich sowohl zum Aussaugen als auch zum Verschlingen (Einsaugen) von Nahrungsobjekten (Crustaceen, Nematoden). (e) Der Pharynx doliiformis ist (vielleicht im Zusammenhang mit seiner terminalen Lage?) hauptsächlich oder ausschließlich ein Schlingpharynx, wobei die durch plötzliches Ơffnen entfaltete Saugwirkung das entscheidende Moment zu sein scheint (vgl. auch LutHER 1962, p. 30).

Diese Kennzeichnungen stimmen auch mit den Befunden von Jennings (1957) an einigen vergleichbaren Arten gut überein. Zur Verdeutlichung sei noch erwähnt: Bei zahlreichen Fütterungsversuchen mit Uteriporus vulgaris und Coelogynopora schulzii, die beide einen Pharynx plicatus besitzen, konnte ich nie ein auch nur teilweises Verschlingen der Oligochaeten beobachten, andererseits schlang Pseudograffilla arenicola mit ihrem kräftigen Pharynx doliiformis einmal selbst einen großen Oligochaeten wenigstens soweit hinunter, wie er nur eben in den Körper paßte, obwohl dann während der Verdauung der größte Teil des Opfers durch die Mundöffnung nach draußen hing.

\section{HERKUNFT DER NAHRUNG}

Wir haben eingangs die Frage aufgeworfen, ob die Nahrungsobjekte der Salzwiesenturbellarien vorwiegend autochthoner oder allochthoner Herkunft sind. Da wir das Verhältnis zum Detritus als möglichem Nahrungsobjekt vorläufig nicht klären können, bleibt auch offen, ob von außerhalb der Salzwiesen eingeschwemmte tote organische Substanz, die nicht mehr als Aas bezeichnet werden kann, für die Ernährung der Turbellarien eine wesentliche Rolle spielt. Wie aber steht es mit eingeschwemmtem Aas und wie mit biotopfremden, in den Salzwiesen nicht lebensfähigen Organismen, die bei Überflutungen hereinkommen, beim Fallen des Wasserstandes aber den Rückweg nicht finden?

Die Antwort läßt sich weitgehend aus der Zusammenstellung der Nahrungsbeobachtungen ableiten. Arten, die sich vorwiegend von Nematoden und Oligochaeten ernähren, können in ihrem Bestand von einer Zufuhr solcher Organismen von außerhalb der Salzwiesen nicht abhängen, da biotopfremde Vertreter gerade dieser beiden stark substratgebundenen Tiergruppen bei Uberflutungen nur in geringer Zahl in die Salzwiesen gelangen. Eine Einschwemmung größerer Mengen von Diatomeen erscheint eher möglich. In großer Zahl können auch Copepoden von außen in die Salzwiesen hereinkommen. Das zeigte eine überflutete Formalinfallen-Serie, deren Fangergebnisse Tabelle 2 wiedergibt. Die Zusammensetzung der Fänge aus überwiegend biotopfremden, aus der benachbarten Stillwasserzone stammenden Tieren (vor allem Mesochra lilljeborgi und Tachidius discipes) zeigt eindrucksvoll, in welchen Mengen solche die Oberfläche des Substrats bevorzugenden Formen von außen herangeführt werden können, und es ist kaum zu bezweifeln, daß auch ohne Formalinfallen ein großer Teil 
der eingeschwemmten Gäste in den Salzwiesen zurückgehalten wird, da die aufragenden Sprosse der Gefäßpflanzen wie ein Kamm oder eine Bürste wirken dürften. Hier sind also für Räuber mit geeigneten Nahrungsaufnahmeorganen (Pharynx rosulatus, vgl. p. 609/610 u. 607 oben) zeitweise geradezu luxuriöse Nahrungsbedingungen gegeben.

\section{Tabelle 2}

Copepoden aus Formalinfallen. Fangplatz: Bottsand (Kieler Bucht). Fangzeit: 10. bis 20. August 1954. (Die Determination der Arten verdanke ich Dr. W. NoodT, Zoologisches Institut, Universität Kiel)

\begin{tabular}{|c|c|c|c|c|c|}
\hline \multirow{2}{*}{$\begin{array}{l}\text { Vegetationszone } \\
\text { Niveauhöhe in } \mathrm{cm} \text { über NN } \\
\text { Uberflutungsdauer in Stunden }\end{array}$} & \multicolumn{3}{|c|}{ Puccinellietum maritimae } & \multicolumn{2}{|c|}{ Juncetum gerardii } \\
\hline & $\begin{array}{r}12 \\
100\end{array}$ & $\begin{array}{l}15 \\
80\end{array}$ & $\begin{array}{l}23 \\
40\end{array}$ & $\begin{array}{l}26 \\
20\end{array}$ & $\begin{array}{r}34 \\
1-2\end{array}$ \\
\hline $\begin{array}{l}\text { Begleiter und Gäste } \\
\text { Cletocamptus confluens (ScHMEIL 1894) }\end{array}$ & & & & & \\
\hline $\begin{array}{l}\text { Cletocamptus confluens (SCHMEIL 1894) } \\
\text { Paronychocamptus nanus (SARS 1908) }\end{array}$ & 2 & $\overline{-}$ & $\overline{4}$ & - & $\overline{-}$ \\
\hline Halicyclops magniceps LILLJEBORG 1901 & 6 & - & 4 & _- & - \\
\hline Microartbridion littorale (POPPE 1881) & 9 & - & 6 & - & - \\
\hline Ectinosoma curticorne BOECK 1872 & - & - & 9 & - & - \\
\hline Halicyclops neglectus KIEFER 1935 & 36 & 3 & 8 & - & - \\
\hline Mesochra lilljeborgi Вовск 1865 & 45 & 96 & 46 & 97 & 60 \\
\hline Tachidius discipes GIESBRECHT 1881 & 100 & 66 & 115 & 20 & 96 \\
\hline Nitocra spinipes BoEck 1864 & 19 & 84 & 9 & 9 & 4 \\
\hline $\begin{array}{l}\text { Charakterarten } \\
\text { Horsiella trisetosa K }\end{array}$ & & & & & \\
\hline Horsiella trisetosa KunZ 1935 & $\overline{5}$ & 二 & 2 & - & - \\
\hline $\begin{array}{l}\text { Parepactophanes minuta KUNZ } 1935 \\
\text { Sigmatidium minor (KuNZ 1935) }\end{array}$ & $\stackrel{3}{-}$ & - & 1 & - & - \\
\hline Schizopera clandestina (KLIE 1924) & - & - & 1 & - & - \\
\hline Schizopera pratensis NoodT 1958 & - & 4 & - & - & - \\
\hline Zahl der determinierten Individuen & & & 206 & 128 & 160 \\
\hline Gesamtza & 7000 & 20000 & 3700 & 2800 & 600 \\
\hline
\end{tabular}

Über die Aufnahme von Aas (allochthon in den Salzwiesen z. B. tote Polychaeten und verendete Fische) läßt sich nur soviel sagen, daß Uteriporus vulgaris und Coelogynopora schulzii auch verendete Oligochaeten annehmen, sofern sie noch nicht zu stark zersetzt sind. Das entspricht den Angaben von Jennings über Polycelis cornuta Schmanda (Seriata, Tricladida maricola; Pharynx plicatus) und über Mesostoma tetragonum O. F. Müller (Neorhabdocoela, Typhloplanoida; Pharynx rosulatus), während der gleiche Autor für Convoluta paradoxa Oerstedt (Acoela; Pharynx simplex) und Macrostomum sp. (Macrostomida; Pharynx simplex) ausdrücklich betont, daß sie nur lebende Organismen annehmen.

Von besonderer Bedeutung ist ferner die Frage nach den Beziehungen zur aerischen Fauna. Die Zusammenstellung der bisher bekannten Nahrungsobjekte ergibt für eine positive Antwort wenig Anhaltspunkte. Zwar müssen wir mit Dipterenlarven als Nahrung von Salzwiesenturbellarien rechnen, doch wissen wir Näheres nicht. Einen Hinweis geben aber doch die Fütterungsexperimente mit Uteriporus vulgaris, der einzigen Triclade unter den Salzwiesenturbellarien (s. BiLIo 1964 b, p. 536). Sie zeigen, daß selbst Schmetterlingsraupen - im Gegensatz zu Copepoden und Nematoden - von dieser Art nicht verschmäht werden, sofern die Kutikula der Raupen nur an irgend- 
einer Stelle vom Pharynx des Turbellars durchbrochen werden kann. Die Schmetterlingsraupen wurden in den Experimenten allerdings ins Wasser der Zuchtschalen gelegt. Die Frage, ob die Salzwiesenturbellarien als Wassertiere (nur Uteriporus scheint sich auch in wasserdampfgesättigter Luft aufhalten zu können, vgl. BiLıo 1964 b) auch Tiere aus lufterfüllten Teilen der Bodenlücken als Nahrung nutzen können, solange diese dort noch aktiv und nicht schon abgestorben und vielleicht infolgedessen bereits benetzbar sind, beantworten diese Experimente also nicht. Es erscheint aber möglich, daß durch längere Überflutungen geschädigte Lufttiere im Wasser von Turbellarien angegriffen und ausgesaugt werden können.

\section{HAUFIGKEIT DER NAHRUNGSORGANISMEN}

Wenn die Turbellarien der Salzwiesen zum größten Teil auf autochthone Nahrungsobjekte angewiesen sind, erhebt sich die Frage nach ihrem zahlenmäßigen Verhältnis zu diesen Nahrungsobjekten. Zur Beantwortung dieser Frage wurde zunächst geprüt, ob große Besiedlungsdichten von Turbellarien mit großen Besiedlungsdichten von Nematoden und Oligochaeten gekoppelt sind. Beim reinen Vergleichen der Zahlen (statistische Berechnungen wurden noch nicht durchgeführt) war kein Zusammenhang

Tabelle 3

Individuendichte der Begleitfauna (Exemplare $100 \mathrm{~cm}^{2} \cdot 3 \mathrm{~cm}$ Boden)

\begin{tabular}{|c|c|c|c|}
\hline Tiergruppe & Minimum & Maximum & Mittelwert \\
\hline & \multicolumn{3}{|c|}{$\begin{array}{l}25 \text { Proben mit mindestens } 40 \text { Turbellarien pro Bezugseinheit }{ }^{1} \\
\text { Herkunft: südwestniederld. Delta-Gebiet }\end{array}$} \\
\hline Turbellarien & 40 & 398 & 108 \\
\hline Nem & 20 & 7800 & 1490 \\
\hline Oligochaeten & 20 & 1500 & 410 \\
\hline & \multicolumn{3}{|c|}{13 Proben von der Nord- und Ostseeküste Schleswig-Holsteins } \\
\hline Dipteren-Larven & & 127 & 38 \\
\hline
\end{tabular}

erkennbar. In Tabelle 3 sind für die 25 berücksichtigten Proben die Minima, Maxima und Mittelwerte wiedergegeben. Der Mittelwert für die Turbellarien (108) erscheint gegenüber den Mittelwerten für die Nematoden (1490) und Oligochaeten (410) für ein Räuber-Beute-Verhältnis recht hoch. Es ist dabei jedoch zu bedenken, daß der Mittelwert für die Turbellarien bei gleicher Zufallsstreuung wie für die beiden anderen Gruppen (also bei Einbeziehung auch der Proben mit weniger als 40 Turbellarien pro $100 \mathrm{~cm}^{2} \times 3 \mathrm{~cm}$ Boden) wesentlich niedriger liegen dürfte. Um einen Vergleich mit den als Nahrungsobjekte der Turbellarien noch problematischen Dipterenlarven zu ermöglichen, sind für diese Gruppe die Werte von 13 Proben aus Norddeutschland hinzugefügt. 


\section{KONKURRENTEN UND FEINDE}

Haben die Salzwiesenturbellarien als Diatomeenfresser und Räuber wichtige Nahrungskonkurrenten? Eine genaue Analyse der Nahrungsbeziehungen auch der anderen im Salzwiesenboden vertretenen Tiergruppen würde den Rahmen dieser Darstellung überschreiten. Betrachten wir noch einmal die Liste der Begleitfauna (Tabelle 1). Die wichtigsten Konkurrenten nach Arten- und Individuenzahl dürften die Nematoden sein. Allein zehn der in den Salzwiesen der schleswig-holsteinischen Küsten festgestellten Nematodenarten (Bilio 1966 a) gehören nach Wirsers Analyse der Beziehungen zwischen Mundhöhlengestalt und Ernährungsweise bei Nematoden (WIESER 1953) zur Gruppe $2 \mathrm{~B}$, in der hauptsächlich Räuber vereinigt sind. Kaum weniger groß dürfte die Bedeutung der Nematoden als Diatomeenfresser sein. Wie weit im übrigen zum Beispiel die Halacariden als Nahrungskonkurrenten der Salzwiesenturbellarien in Frage kommen, läßt sich vorläufig noch nicht übersehen. Als Besonderheit sei erwähnt, daß (nach einer Beobachtung beim Lebendsortieren der Fauna) auch Ciliaten sich gelegentlich Nematoden einverleiben können, obwohl es normalerweise eher umgekehrt sein dürfte. Allerdings war im beobachteten Fall der Nematode so lang, daß er im Inneren des Einzellers einen Knick erhielt. Dieser machte ihn so sperrig, daß die Ciliaten-Pellikula an der Knickstelle stark ausgebuchtet wurde und (vielleicht unter zusätzlichem Deckglasdruck) schließlich durchbrach.

Über die Feinde der Salzwiesenturbellarien lassen sich etwas nähere Angaben machen. Soweit sie nicht unter den Turbellarien selbst zu suchen sind, gehören sie einerseits als übergeordnete Räuber zu den Polychaeten und andererseits als Parasiten zu den Sporozoen. Der einzige Polychaet, der in den Salzwiesen als Turbellarienfresser in Frage kommt, ist Nereis diversicolor. Eine Reihe von Fütterungsversuchen mit Turbellarien verschiedener Verwandtschaftsgruppen verlief positiv. Nereis diversicolor scheint jedoch der einzige den Salzwiesenturbellarien übergeordnete Räuber von Bedeutung zu sein. Denn andere räuberische Wassertiere geeigneter Größe fehlen in den Salzwiesen, wenn man von Dipterenlarven, die als Turbellarienfresser nicht bekannt sind, absieht. (Die Nemertine Prostoma obscurum ist bisher nur in Salzwiesen der Kieler Bucht angetroffen worden, und auch dort nur an einer einzigen Stelle - wenn auch während des ganzen Jahres.) Über das Verhältnis von räuberischen Formen der aerischen Phase des Lebensraumes, beispielsweise von Spinnen und Käfern, zu Turbellarien wissen wir nichts. Es ist auch zu bedenken, daß angesichts so kleiner Dimensionen, wie sie in den Lückenräumen des Bodens herrschen, die mechanische Schranke, die die Oberflächenspannung des Wassers setzt, als sehr hoch angesehen werden muß, und auch die Wahrnehmung der Beute im anderen Milieu und nicht zuletzt der Salzgehalt erscheinen in diesem Zusammenhang als besonders problematisch. Wir können daher wohl im großen und ganzen feststellen, daß die Nahrungskette in den Salzwiesen bei den Turbellarien endet, sobald Nereis diversicolor fehlt, und das ist unter normalen Bedingungen bereits wenig oberhalb der unteren Salzwiesengrenze der Fall. Zudem ist in Salzwiesen mesohaliner Bereiche, beispielsweise der finnischen Küste, Nereis bisher überhaupt nicht angetroffen worden. 


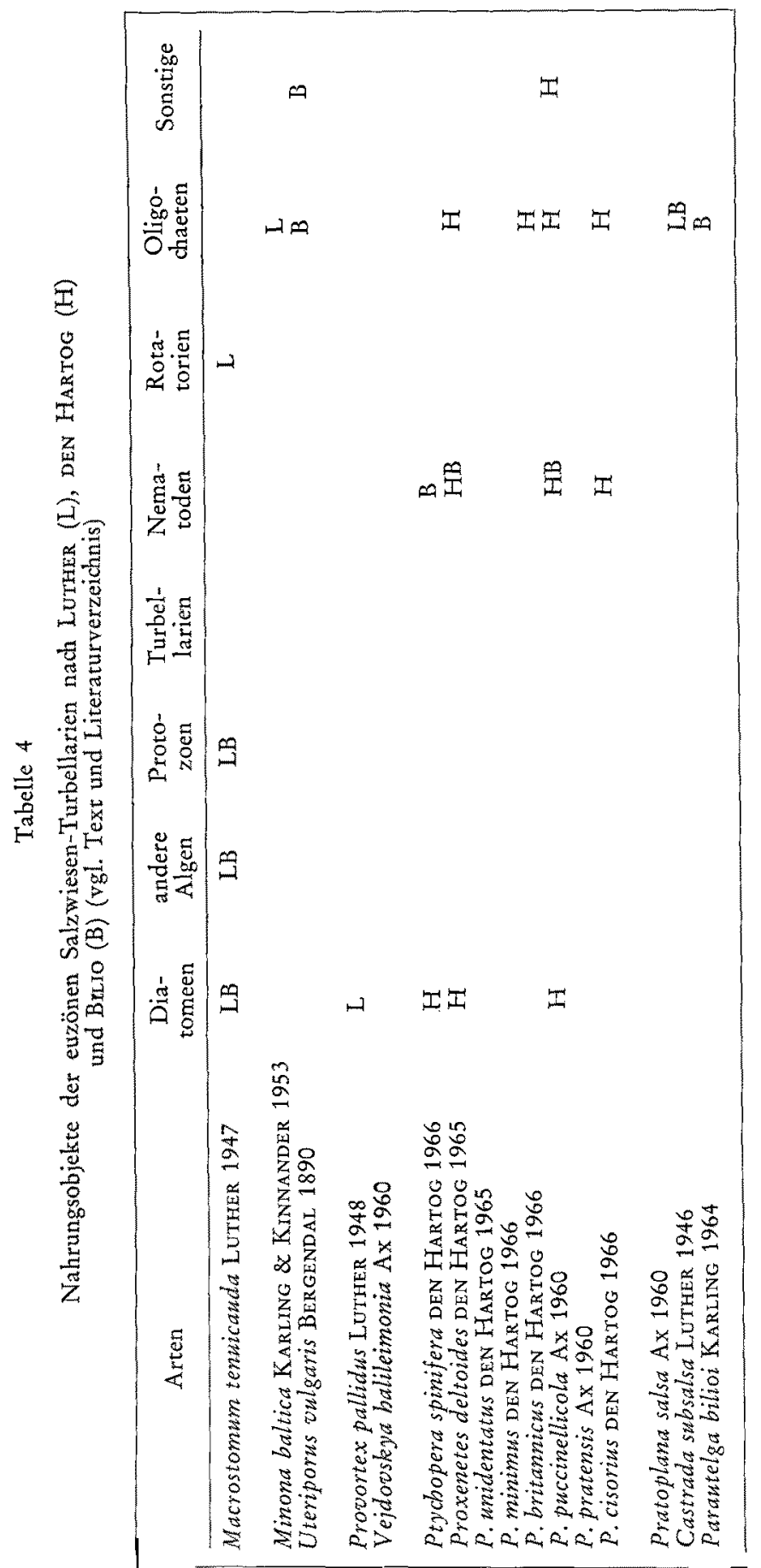


Nahrungsbeziehungen der Salzwiesen-Turbellarien

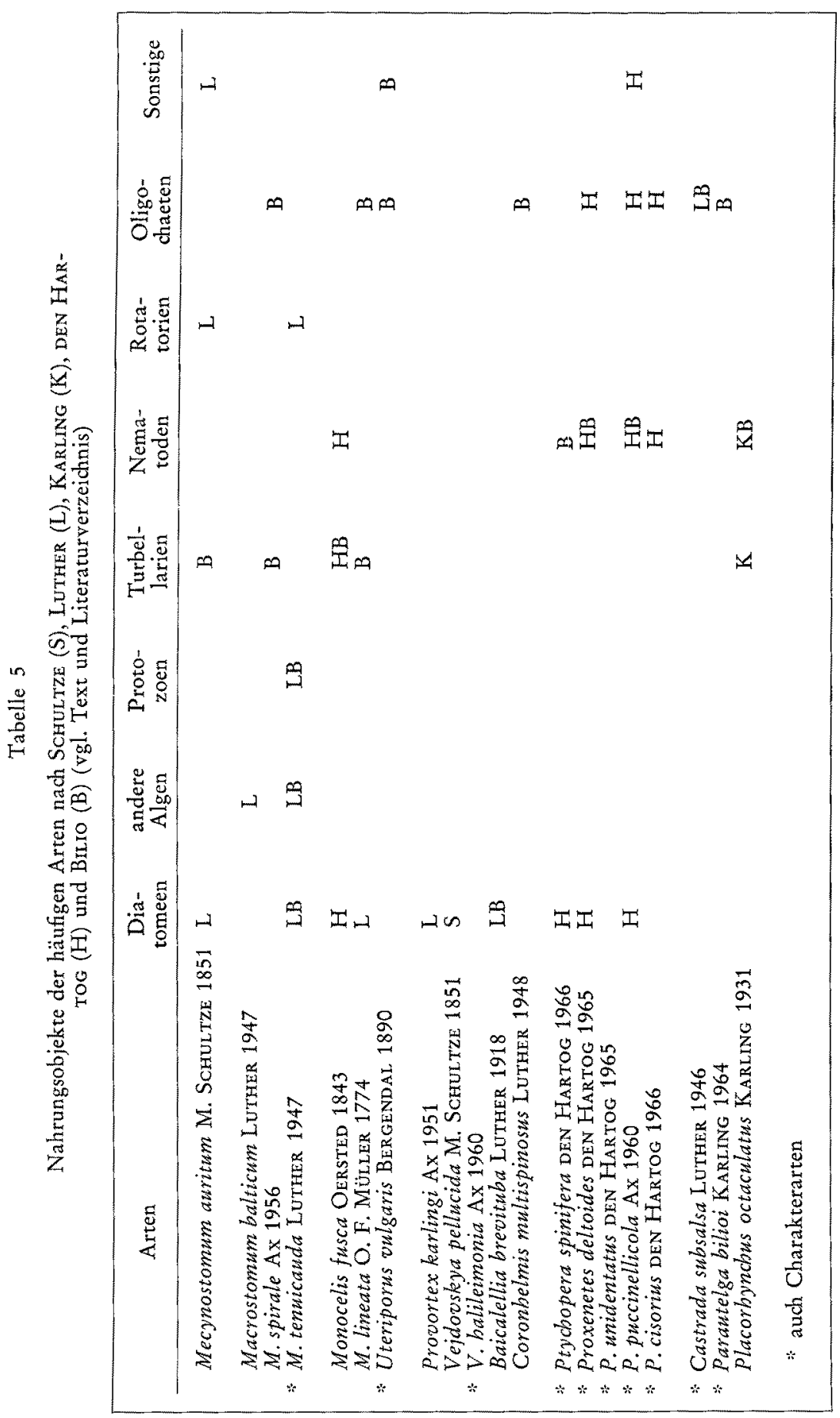




\section{VERGLEICH MIT ANDEREN STRANDBIOTOPEN}

Eine schematische Übersicht über das Gefüge der Nahrungsbeziehungen gibt $\mathrm{Ab}$ bildung 3. Es bleibt uns nach dieser zusammenfassenden Wiedergabe noch die Aufgabe, die Salzwiesen unter dem Gesichtspunkt der Nahrungsbeziehungen mit den anderen Strandbiotopen zu vergleichen. In Tabelle 4 sind alle bekannten Nahrungsobjekte der euzönen Salzwiesen-Turbellarien (= Spezifische und Präferenten, nach TISCHLER

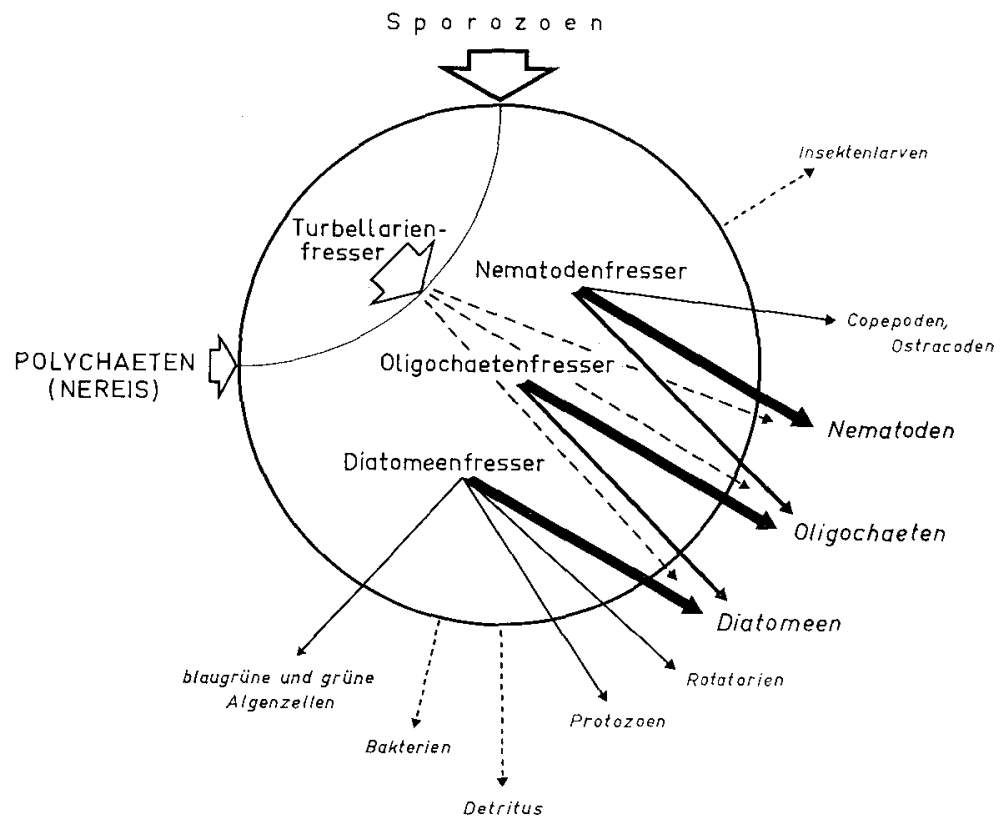

Abb. 3: Gefüge der Nahrungsbeziehungen der Salzwiesen-Turbellarien. Innerhalb des Kreises nur Turbellarien, unten und rechts die Nahrungsobjekte, links Räuber der auf die Turbellarien folgenden trophischen Stufe, oben Parasiten. Die punktierten Linien, die vom Rand des Kreises ausgehen, bezeichnen fragliche Nahrungsbeziehungen

1949) zusammengestellt. Danach überwiegen unter den Nahrungsobjekten der besonders charakteristischen Salzwiesenturbellarien die Oligochaeten. Dieses Bild verschiebt sich deutlich zugunsten der Diatomeen, wenn wir nur nach der Zahl der in den Salzwiesen gefundenen Individuen der Turbellarienarten vorgehen und ausschließlich die häufigen Arten zusammenstellen (Tabelle 5). Zwar sind auch jetzt noch Charakterarten vertreten, doch ist eine Reihe von Arten mit deutlichen Beziehungen zu Biotopen ohne geschlossene Gefäßpflanzendecke hinzugekommen, und zwar - aus der Stillwasserzone in die Salzwiesen hineinreichend - vor allem der spezifische Diatomeenfresser Baicalellia brevitubus und auch die beiden Proseriaten Monocelis lineata und M. fusca. Außerdem zeigt der Vergleich der beiden Tabellen, daß Turbellarien bei den spezifischen Salzwiesenturbellarien als Nahrung völlig fehlen. Die Turbellarienfresser unter den Turbellarien selbst lassen vielmehr eine deutliche Tendenz zur Stillwasserzone er- 
kennen, was besonders auch für die als extremer und relativ großer Räuber nicht zu den häufigen Formen zählende Pseudograffilla arenicola gilt.

Als einziger Vertreter der auf die Turbellarien folgenden trophischen Stufe hat sich in den Salzwiesen Nereis diversicolor erwiesen, und da dieser Polychaet bereits in der untersten Salzwiesenzone seine normale obere Verteilungsgrenze erreicht, können wir in der Vertikal e n des Strandes (s. Abb. 4) in bezug auf die Länge der Nahrungskette einen Gradienten erkennen: Je kleiner mit zunehmender Niveauhöhe die durch wasseraktive Tiere besiedelbaren Aktionsräume werden, um so kürzer wird die Nahrungskette innerhalb des aquatischen Milieus. Dieser Gradient wird natürlich am deutlichsten erkennbar, wenn man das angrenzende Meer in die Betrachtung einbezieht und vom Meer aus über die Gezeitenzone zum eigentlichen Strand fortschreitet: Zuerst bleiben die großen Fische weg, die die kleinen Fische fressen, dann scheiden die kleinen

Tabelle 6

Nereis diversicolor O. F. MülleR 1776 aus Formalinfallen. Fangplatz: Bottsand (Kieler Bucht). Fangzeit: 16. Dezember 1954 bis 20. Januar $1955(\mathrm{n}, \mathrm{u})$ beziehungsweise 16. Dezember 1954 bis 28. Januar $1955(\mathrm{~m}, \mathrm{q}, \mathrm{z})$

\begin{tabular}{|lccccc|}
\hline & \multicolumn{3}{c}{ Puccinellietum } & maritimae & \multicolumn{2}{c|}{ Juncetum gerardii } \\
Vegetationszone & $\mathrm{z}$ & $\mathrm{u}$ & $\mathrm{q}$ & $\mathrm{n}$ & $\mathrm{m}$ \\
Fallenstandort & 12 & 15 & 23 & 26 & 34 \\
Niveau in cm über NN & 74 & 59 & 63 & 15 & 16 \\
\hline Individuenzahl & & & & & \\
\hline
\end{tabular}

Fische aus, die Polychaeten fressen, darauf die Polychaeten, und in den oberen Bereichen der Salzwiesen schließlich die Turbellarien selbst. Daß die Nahrungskette zeitweise allerdings auch oberhalb der untersten Salzwiesenzone bis zu den Polychaeten reichen kann, zeigen Überflutungsfänge von Formalinfallen (vgl. Tabelle 6): Nereis diversicolor ist nach diesen Ergebnissen offensichtlich in der Lage, bei länger anhaltendem Hochwasser wandernd bis in das Juncetum gerardii vorzudringen.

In der Horizontalen des Strandes sind die Besiedlungsdichten im Zusammenhang mit der Biomasse der Primärproduzenten von besonderem Interesse. Aus dem Stranddiagramm in der in Abbildung 4 wiedergegebenen Form läßt sich ablesen, daß die vorhandene Biomasse vom Brandungsstrand zum Salzwiesenufer erheblich zunimmt. Zum Vergleich sind für alle drei Strandabschnitte die bisher bekannten maximalen Individuenzahlen der Turbellarien pro $100 \mathrm{~cm}^{2}$ Fläche und $3 \mathrm{~cm}$ Tiefe ermittelt und in das Diagramm eingetragen worden. Das Ergebnis war zunächst eine Uberraschung, läßt sich doch jetzt ein entgegengesetzt gerichteter Gradient erkennen. Berücksichtigt man jedoch die Milieubedingungen und die Nahrungsbeziehungen, so wird dieses Ergebnis besser verständlich. Am lotischen Strand, und hier wieder besonders in der eigentlichen Brandungszone, sind die Lebensbedingungen für Primärproduzenten (vor allem für sessile Pflanzen) zwar sehr ungünstig, Plankton hingegen und aufgewirbelte oder losgerissene Organismen aus den benachbarten tiefer gelegenen Benthalzonen werden in großer Zahl herangetragen und von den sich brechenden Wellen verletzt oder zerschlagen. Für Räuber und Aasfresser sind die Ernährungsbedingungen also optimal, sofern sie nur mit den extremen mechanischen Bedingungen des Milieus 
zurechtkommen. Das aber gilt für eine Reihe von Vertretern einer bestimmten systematischen Gruppe von Turbellarien, nämlich der Otoplaniden, in besonderem Maße, und nach ihnen wird daher diese Zone auch Otoplanen-Zone genannt.

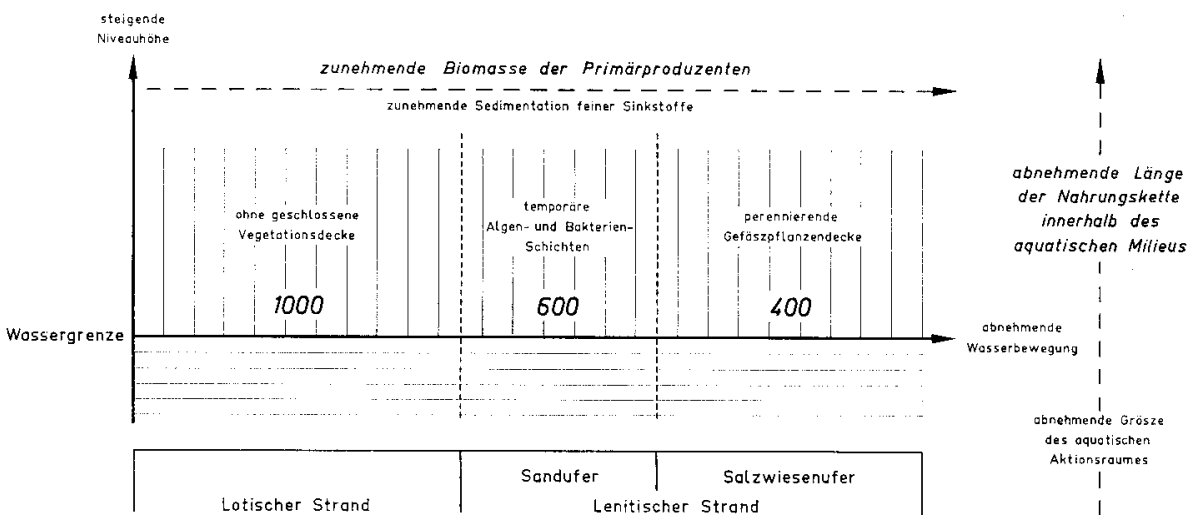

Abb. 4: Maximale Individuendichten der Turbellarien (Exemplare pro $100 \mathrm{~cm}^{2} \cdot 3 \mathrm{~cm}$ Boden) im Wasserrandbereich verschiedener Strandabschnitte. Werte für den lotischen Strand nach Ergebnissen von STERrer (1965, p. 215) von der finnischen Küste (Originalprobengröße: $1 \mathrm{dm}^{3}$ ), für den lenitischen Strand (nach eigenen Ergebnissen) aus dem südwestniederländischen Delta.. Gebiet (Größe der berücksichtigten Proben: $50 \mathrm{~cm}^{2} \cdot 3 \mathrm{~cm}$ ). Hinsichtlich der Strandeinteilung vgl. Brilo (1964 a, Fig. 3, und 1965, Fig. 1)

Während für die große Individuendichte der Turbellarien am Wasserrand des lotischen Strandes weder die vorhandene Biomasse der Primärproduzenten noch die Primärproduktion pro Zeiteinheit ausschlaggebend sein kann, erscheint es nicht ausgeschlossen, daß am Streifensandufer (= Sandufer des lenitischen Strandes) die Primärproduktion der Kleinalgen zeitweise die Grundlage für eine besonders große Individuendichte der Turbellarien bietet. In den Salzwiesen könnte die Primärproduktion der Kleinalgen infolge der Beschattung durch die Gefäßpflanzen hinter der am Streifensandufer zurückbleiben. Aufschluß darüber können jedoch erst weitere Untersuchungen bringen.

\section{ZUSAMMENFASSUNG}

1. Die Nahrung der Salzwiesenturbellarien besteht vorwiegend aus Oligochaeten, Nematoden und Diatomeen. Für einzelne Arten (Mecynostomum, Pseudograffilla) spielen auch andere Turbellarien als Nahrung eine erhebliche Rolle. Nachgewiesene Nahrungsobjekte sind ferner Copepoden, Rotatorien, Ciliaten, Thekamöben und grüne Einzeller.

2. Den Hauptanteil am Nahrungsspektrum der für lenitische Uferbiotope besonders charakteristischen Proxenetes- und Ptychopera-Arten haben die Nematoden, die andererseits bei der einzigen Acoelen und auch bei den Seriaten völlig zurücktreten. 
3. Auf Grund der Nahrungsbeobachtungen an Salzwiesenturbellarien lassen sich der Pharynx plicatus als Saug- und Schlürfpharynx, der Pharynx rosulatus als Saugund Schlingpharynx und der Pharynx doliiformis als Schlingpharynx charakterisieren, während der Pharynx simplex als sehr wenig spezialisiert erscheint.

4. Die Nahrungsobjekte stammen hauptsächlich aus den Salzwiesen selbst, eingeschwemmte Organismen (z. B. Copepoden) können jedoch zeitweise die Ernährungsbasis erweitern. Aas können Coelogynopora schulzii und die Triclade Uteriporus vulgaris verwerten.

5. Nach den Ergebnissen von Fütterungsexperimenten kann Uteriporus auch unter Wasser geratene luftlebende Insektenlarven (Schmetterlingsraupen) angreifen und aussaugen.Für eine Aufnahme luftlebender Tiere direkt aus den Lufträumen des Bodens liegen keine Anhaltspunkte vor.

6. Ein Zusammenhang zwischen großen Individuendichten von Turbellarien einerseits und von Oligochaeten und Nematoden andererseits ist im Einzelfall nicht erkennbar, die mittleren Individuendichten der Oligochaeten und Nematoden jedoch liegen für turbellarienreiche Proben um das rund Vier- beziehungsweise Vierzehnfache höher als die mittlere Individuendichte der Turbellarien.

7. Als wichtigste Nahrungskonkurrenten kommen die Räuber und Diatomeenfresser unter den Nematoden in Frage; von den in Salzwiesen deutscher Küsten festgestellten Nematodenarten sind etwa zehn zu den Räubern zu rechnen.

8. $\mathrm{Zu}$ den Feinden gehören einerseits als Räuber der Polychaet Nereis diversicolor und einige Arten der Turbellarien selbst, andererseits als Parasiten Vertreter der Sporozoen.

9. Während die aquatische Nahrungskette in der untersten Salzwiesenzone eu- und polyhaliner Bereiche der Nord- und Ostsee noch bis zu Nereis reicht, findet sie auf etwas höherem Niveau bereits bei den Turbellarien ihr Ende. Im Verlauf länger anhaltender Überflutungen kann der Polychaet allerdings bis ins Juncetum gerardii vordringen.

10. Obwohl die Biomasse der Primärproduzenten am Brandungsstrand weit hinter der am Salzwiesenufer zurückbleibt, ist am Brandungsstrand eine erheblich größere maximale Individuendichte der Turbellarien festgestellt worden als am Salzwiesenufer. Die Grundlage für die hohe Dichte am Brandungsstrand bildet wahrscheinlich die reiche Zufuhr sowohl noch lebender als auch bereits durch die Wucht der Brandung getöteter Nahrungsorganismen aus dem Plankton und aus den benachbarten tiefer gelegenen Benthalzonen.

Die finanzielle Möglichkeit zur Fortführung meiner Untersuchungen über die Mikrofauna der lenitischen Strandbiotope verdanke ich der „Nederlandse Organisatie voor Zuiver-Wetenschappelijk Onderzoek (ZWO) “, die mir auf Antrag von Herrn Dr. K. F. VAAs, Direktor der Abteilung Delta-Untersuchungen des Hydrobiologischen Instituts der Königlichen Niederländischen Akademie der Wissenschaften, ein Gastforscherstipendium gewährt. Die neuen Daten über die Nahrung der Turbellarien konnte ich teils in Deutschland während meiner Arbeit als Stipendiat der Deutschen Forschungsgemeinschaft im Zoologischen Institut der Universität Kiel sammeln, teils in Finnland als Gast der Zoologischen Station Tvärminne, und ferner in den Niederlanden während meiner Aufenthalte am Delta-Institut in Yerseke. Allen Förderern meiner Arbeit und den Freunden und Helfern in den genannten Instituten fühle ich mich für ihr Wohlwollen zu großem Dank verpflichtet. 


\section{ZITIERTE LITERATUR}

Ax, P., 1961. Verwandtschaftsbeziehungen und Phylogenie der Turbellarien. Ergbn. Biol. 24, $1-68$.

BiLIo, M., 1964a. Die biozönotische Stellung der Salzwiesen unter den Strandbiotopen. Zool. Anz. (Suppl. Bd) 27, 417-425.

- 1964b. Die aquatische Bodenfauna von Salzwiesen der Nord- und Ostsee. I. Biotop und ökologische Faunenanalyse: Turbellaria. Int. Revue ges. Hydrobiol. 49, 509-562.

- 1965. Die Verteilung der aquatischen Bodenfauna und die Gliederung der Vegetation im Strandbereich der deutschen Nord- und Ostseeküste. Botanica gothoburg. 3, $25-42$.

- 1966a. Die aquatische Bodenfauna von Salzwiesen der Nord- und Ostsee. II. Okologische Faunenanalyse: Hydrozoa, Nematodes, Rotatoria, Gastrotricha, Nemertini, Polychaeta Oligochaeta, Halacaridae, Ostracoda, Copepoda. Int. Revue ges. Hydrobiol. 51, 147-195.

- 1966b. Charakteristische Unterschiede in der Besiedlung finnischer, deutscher und holländischer Küstensalzwiesen durch Turbellarien. Veröff. Inst. Meeresforsch. Bremerh. (Sonderbd) 2, 305-317.

Hartog, C. Den, 1964a. Proseriate flatworms from the deltaic area of the rivers Rhine, Meuse and Scheldt. 1-2. Proc, K. ned. Akad. Wet. (C) 67, 10-34.

- 1964b. A preliminary revision of the Proxenetes group (Trigonostomidae, Turbellaria). 1-3. Proc. K. ned. Akad. Wet. (C) 67, 371-407.

- 1965a-b. A preliminary revision of the Proxenetes group (Trigonostomidae, Turbellaria). 4-5. Proc. K. ned. Akad. Wet. (C) 68, 98-111; 112-120.

- 1966a-b. A preliminary revision of the Proxenetes group (Trigonostomidae, Turbellaria). 6-10 + Suppl. Proc. K. ned. Akad. Wet. (C) 69, 97-163;557-570.

Jennings, J. B. 1957. Studies on feeding, digestion, and food storage in free-living flatworms (Plathyhelminthes: Turbellaria). Biol. Bull. mar. biol. Lab., Woods Hole 112, 63-80.

KarLING, T. G., 1956. Zur Kenntnis einiger Gnathorhynchiden nebst Beschreibung einer neuen Gattung. Ark. Zool. 9, 343-353.

- 1963. Die Turbellarien Ostfennoskandiens. V. Neorhabdocoela. 3. Kalyptorhynchia. Fauna fenn. 17, 1-59.

LUTHER, A., 1948. Untersuchungen an rhabdocoelen Turbellarien. VII. Uber einige marine Dalyellioida. VIII. Beiträge zur Kenntnis der Typhloplanoida. Acta zool. fenn. 55, 1-122.

- 1960. Die Turbellarien Ostfennoskandiens. I. Acoela, Catenulida, Macrostomida, Lecithoepitheliata, Prolecithophora und Proseriata. Fauna fenn. 7, 1-154.

- 1962. Die Turbellarien Ostfennoskandiens. III. Neorhabdocoela. 1. Dalyellioida, Typhloplanoida: Byrsophlebidae und Trigonostomidae. Fauna fenn. 12, 1-71.

- 1963. Die Turbellarien Ostfennoskandiens. IV. Neorhabdocoela. 2. Typhloplanoida: Typhloplanidae, Solenopharyngidae und Carcharodopharyngidae. Fauna fenn. 16, 1-160.

Schultze, M. S., 1851. Beiträge zur Naturgeschichte der Turbellarien. Koch, Greifswald, 78 pp.

STERrer, W., 1965. Zur Okologie der Turbellarien eines südfinnischen Sandstrandes. Botanica gothoburg. 3, 211-219.

TrsCHLER, W., 1949. Grundzüge der terrestrischen Tierökologie. Vieweg, Braunschweig, 220 pp.

Westrbad, E., 1923. Zur Physiologie der Turbellarien. I. Die Verdauung. II. Die Exkretion. Acta Univ. Lund. 33 (6), 4-212.

- 1955. Marine "Alloeocoels" (Turbellaria) from North Atlantic and Mediterranean coasts. I. Ark. Zool. 7, 491-526.

Wieser, W., 1953. Die Beziehung zwischen Mundhöhlengestalt, Ernährungsweise und Vorkommen bei freilebenden marinen Nematoden; eine ökologisch-morphologische Studie. Ark. Zool. 4, 439-484. 


\section{Diskussion im Anscbluß an den Vortrag BıLıo}

Brattström: Können Vögel als Turbellarienfresser in den Strandwiesen auftreten?

Brıro: Bisher gibt es dafür keine Anhaltspunkte. Im allgemeinen dürften die Salzwiesen-Turbellarien für Vögel als Nahrungsorganismen zu klein sein: Nur sechs von den 50 Arten erreichen eine Länge von $5 \mathrm{~mm}$, und nur eine einzige davon - die extrem dünne Coelogynopora schulzii - kann mehr als $1 \mathrm{~cm}$ lang werden.

Amanieu: M. Bitio a-t-il observé une répartition des Turbellariés qu'il a étudiés, caractéristique des différents biotopes des près salés? En particulier la faunule du Salicornieto-Spartinetum est-elle très différente de celle des flaques supralittorales du schorre supérieur?

Bilio: Ja, je nach Struktur und Wassergehalt des Bodens zeigt auch die Artenkombination der Turbellarien deutliche Unterschiede zwischen verschiedenen Salzwiesentypen und -zonen, besonders zwischen den feinmineralreichen Böden der vorwiegend durch Sedimentation verlandenden Gebiete und den zur Vertorfung neigenden Böden der hauptsächlich durch Pflanzenwuchs über das Meeresniveau hinauswachsenden Küstenabschnitte. Unterschiede zwischen verschiedenen Niveauhöhen äußern sich vor allem im Anteil der Meerestiere einerseits und der Brackwassertiere andererseits, wobei die Brackwassertiere zugleich eine stärkere Tendenz zu Interstitialbedingungen erkennen lassen. Unsere Untersuchungen galten bisher in erster Linie dem Puccinellietum maritimae und dem Juncetum gerardii; die eher als Röhrichte denn als Wiesen anzusprechenden Spartina-Gesellschaften wurden nur gelegentlich berïcksichtigt. 